\title{
Modified Linear Theory for Spinning or Non-Spinning Projectiles
}

\author{
D.N. Gkritzapis*,1, E.E. Panagiotopoulos ${ }^{2}$, D.P. Margaris ${ }^{3}$ and D.G. Papanikas ${ }^{4}$
}

\author{
${ }^{1}$ Laboratory of Firearms and Tool Marks Section, Criminal Investigation Division, Hellenic Police, and Post graduate \\ Student, Mechanical Engineering and Aeronautics Department, University of Patras, Greece \\ ${ }^{2}$ Post-graduate Student, Mechanical Engineering and Aeronautics Department, University of Patras, Greece \\ ${ }^{3}$ Professor, Mechanical Engineering and Aeronautics Department, University of Patras, Greece \\ ${ }^{4}$ Ex-Professor, Mechanical Engineering and Aeronautics Department, University of Patras, Greece
}

\begin{abstract}
Static and dynamic stability are the most important phenomena for stable flight atmospheric motion of spin and fin stabilized projectiles. If the aerodynamic forces and moments and the initial conditions are accurately known, an essentially exact simulation of the projectile's synthesized pitching and yawing motion can be readily obtained by numerical methods. A modified trajectory linear theory of the same problem implies an approximate solution.
\end{abstract}

\section{INTRODUCTION}

More than 80 years ago, English ballisticians [1] constructed the first rigid six-degree-of-freedom projectile exterior ballistic model. Their model contained a reasonably complete aerodynamic force and moment expansion for a spinning shell and included aerodynamic damping along with Magnus force and moment. Guided by an extensive set of yaw card firings, these researchers also created the first approximate analytic solution of the six-degree-of-freedom projectile equations of motion by introducing a set of simplifications based on clever linearization by artificially separating the dynamic equations into uncoupled groups. The resulting theory is commonly called projectile linear theory. Kent [2], Neilson and Synge [3], Kelley and McShane [4], and Kelley et al. [5] made refinements and improvements to projectile linear theory.

Projectile linear theory has proved an invaluable tool in understanding basic dynamic characteristics of projectiles in atmospheric flight, for establishing stability criteria for finand spin-stabilized projectiles, and for extracting projectile aerodynamic loads from spark range data.

In the present work, the full six degrees of freedom (6DOF) projectile flight dynamics atmospheric model is considered for the accurate prediction of short and long range trajectories of high spin and fin-stabilized projectiles. It takes into consideration the influence of the most significant forces and moments, in addition to gravity.

Projectiles, which are inherently aerodynamically unstable, can be stabilized with spin. For this condition, the spin rate must be high enough to develop a gyroscopic moment, which overcomes the aerodynamic instability, and the projectile is said to be gyroscopically stable. This is the case for the most gun launched projectiles (handguns, rifles, cannons,

*Address correspondence to this author at the Laboratory of Firearms and Tool Marks Section, Criminal Investigation Division, Hellenic Police, and Post graduate Student, Mechanical Engineering and Aeronautics Department, University of Patras, Greece; E-mail: gritzap@yahoo.gr etc.) where the rifling of the barrel provides the required axial spin to projectile. In describing this condition, a gyroscopic stability factor can be applied, which is obtained from the roots of the modified linear theory in the equations of projectile motion.

Also, dynamic stability is defined as the condition where a system is perturbed and the ensuing oscillatory has a tendency to either decrease or increase. Note that this definition assumes that the static stability is present, otherwise the oscillatory motion would not occur.

\section{PROJECTILE MODEL}

The present analysis considers two different types of representative projectiles: a spin-stabilized of $105 \mathrm{~mm}$ and a mortar fin-stabilized of $120 \mathrm{~mm}$.

Basic physical and geometrical characteristics data of the above-mentioned $105 \mathrm{~mm} \mathrm{HE} \mathrm{M1} \mathrm{and} \mathrm{the} \mathrm{non-rolling,}$ finned $120 \mathrm{~mm} \mathrm{HE}$ mortar projectiles are illustrated briefly in Table $\mathbf{1 .}$

Table 1. Physical and Geometrical Data of $105 \mathrm{~mm}$ and $120 \mathrm{~mm}$ Projectiles Types

\begin{tabular}{|c|c|c|}
\hline Characteristics & $\begin{array}{c}\mathbf{1 0 5} \text { mm HE M1 } \\
\text { projectile }\end{array}$ & $\begin{array}{c}\mathbf{1 2 0} \text { mm HE mortar } \\
\text { projectile }\end{array}$ \\
\hline \hline Reference diameter, mm & 114.1 & 119.56 \\
\hline Total Length, mm & 494.7 & 704.98 \\
\hline Total mass, kg & 15.00 & 13.585 \\
\hline $\begin{array}{c}\text { Axial moment of inertia, } \\
\text { kg-m }\end{array}$ & $2326 \times 10^{-2}$ & $2335 \times 10^{-2}$ \\
\hline $\begin{array}{c}\text { Transverse moment of } \\
\text { inertia, kg-m }\end{array}$ & $23118 \times 10^{-2}$ & $23187 \times 10^{-2}$ \\
\hline $\begin{array}{c}\text { Centre of gravity from } \\
\text { the base, } \mathrm{mm}\end{array}$ & 113.4 & 422.9 \\
\hline
\end{tabular}




\section{TRAJECTORY FLIGHT SIMULATION MODEL}

Flight mechanics of most projectile configurations can be captured using a rigid body six degrees of freedom dynamic model. The six degrees of freedom flight analysis comprise the three translation components $(\mathrm{x}, \mathrm{y}, \mathrm{z})$ describing the position of the projectile's center of mass and three Euler angles $(\varphi, \theta, \psi)$ describing the orientation of the projectile body with respect to (Fig. 1).

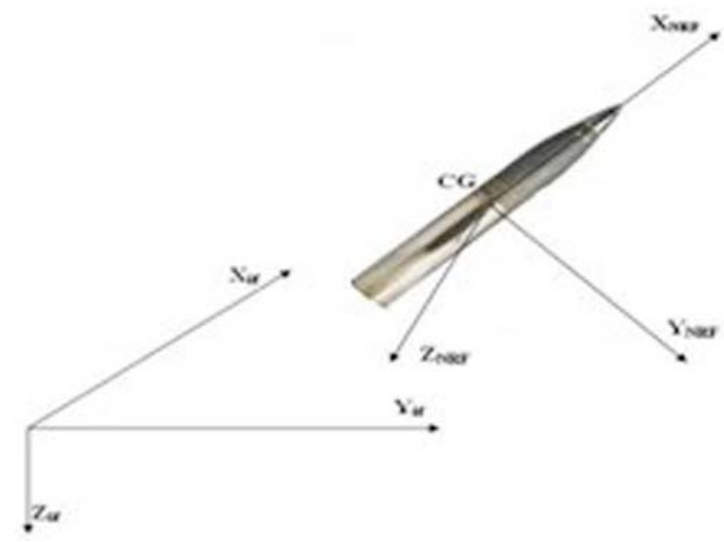

Fig. (1). No-roll (moving) and fixed (inertial) coordinate systems for the projectile trajectory analysis.

Two main coordinate systems are used for the computational approach of the atmospheric flight motion. The one is a plane fixed (inertial frame) at the firing site. The other is a no-roll rotating coordinate system moving with the projectile body (no-roll-frame, NRF, $\varphi=0$ ) with the $X_{\mathrm{NRF}}$ axis along the projectile axis of symmetry and $\mathrm{Y}_{\mathrm{NRF}}, \mathrm{Z}_{\mathrm{NRF}}$ axes oriented so as to complete a right hand orthogonal system.

Newton's laws of the motion state that the rate of change of linear momentum must equal the sum of all the externally applied forces (1) and the rate of change of angular momentum must equal the sum of the externally applied moments (2), respectively.

$m \frac{d \vec{V}}{d t}=\sum \vec{F}+m \vec{g}$

$\frac{d \vec{H}}{d t}=\sum \vec{M}$

The total force acting on the projectile comprises the weight, the aerodynamic force and the Magnus force. The total moment acting on the projectile comprises the moment due to the standard aerodynamic force, the Magnus aerodynamic moment and the unsteady aerodynamic moment. The dynamic equations of motion [6-9] are derived in the nonrolling frame and provided in equations (3) up to (6):

$\left\{\begin{array}{l}\bar{x}_{i f} \\ \bar{y}_{i f} \\ \bar{z}_{i f}\end{array}\right\}=\left[\begin{array}{ccc}\cos \theta \cos \psi & -\sin \psi & \sin \theta \cos \psi \\ \cos \theta \sin \psi & \cos \psi & \sin \theta \sin \psi \\ -\sin \theta & 0 & \cos \theta\end{array}\right]\left\{\begin{array}{c}\tilde{u}_{N R F} \\ \tilde{v}_{N R F} \\ \tilde{w}_{N R F}\end{array}\right\}$

for the position of projectile's center of mass and

$\left\{\begin{array}{l}\bar{\phi} \\ \bar{\theta} \\ \bar{\psi}\end{array}\right\}=\left[\begin{array}{ccc}1 & 0 & t_{\theta} \\ 0 & 1 & 0 \\ 0 & 0 & 1 / \cos \theta\end{array}\right]\left\{\begin{array}{l}\tilde{p}_{N R F} \\ \tilde{q}_{N R F} \\ \tilde{r}_{N R F}\end{array}\right\}$ and for the orientation of the flight body with the classical Euler angles $\varphi, \theta, \psi$. From the two laws of Newton's motion the following equations (5) and (6) are derived, respectively:

$$
\begin{aligned}
& \left\{\begin{array}{l}
\overline{\tilde{u}}_{\text {NRF }} \\
\overline{\tilde{v}}_{\text {NRF }} \\
\overline{\tilde{w}}_{\text {NRF }}
\end{array}\right\}=\left\{\begin{array}{l}
\tilde{F} x_{\text {TOTAL }} / m \\
\tilde{F} y_{\text {TOTAL }} / m \\
\tilde{F} z_{\text {TOTAL }} / m
\end{array}\right\}+ \\
& +\left[\begin{array}{ccc}
0 & \tilde{r}_{N R F} & -\tilde{q}_{N R F} \\
-\tilde{r}_{N R F} & 0 & -\tilde{r}_{N R F} t_{\theta} \\
\tilde{q}_{N R F} & \tilde{r}_{N R F} t_{\theta} & 0
\end{array}\right]\left\{\begin{array}{l}
\tilde{u}_{N R F} \\
\tilde{v}_{N R F} \\
\tilde{w}_{N R F}
\end{array}\right\} \\
& \left\{\begin{array}{l}
\tilde{\tilde{p}}_{N R F} \\
\tilde{\tilde{q}}_{N R F} \\
\tilde{\tilde{r}}_{N R F}
\end{array}\right\}=\left[I^{-1}\right]\left[\begin{array}{c}
\left\{\begin{array}{c}
\tilde{L}_{\text {TOTAL }} \\
\tilde{M}_{\text {TOTAL }} \\
\tilde{N}_{\text {TOTAL }}
\end{array}\right\}-\left[\begin{array}{ccc}
0 & -\tilde{r}_{N R F} & \tilde{q}_{N R F} \\
\tilde{r}_{N R F} & 0 & \tilde{r}_{N R F} t_{\theta} \\
-\tilde{q}_{N R F} & -\tilde{r}_{N R F} t_{\theta} & 0
\end{array}\right] \\
{\left[\begin{array}{lll}
I_{X X} & I_{X Y} & I_{X Z} \\
I_{Y X} & I_{Y Y} & I_{Y Z} \\
I_{Z X} & I_{Z Y} & I_{Z Z}
\end{array}\right]\left\{\begin{array}{l}
\tilde{p}_{N R F} \\
\tilde{q}_{N R F} \\
\tilde{r}_{N R F}
\end{array}\right\}}
\end{array}\right.
\end{aligned}
$$

The total force acting on the projectile in equation (5) comprises the weight $W_{f}$, the aerodynamic force $A_{f}$ and Magnus force $M_{f}$ :

$$
\left\{\begin{array}{l}
\tilde{F} x_{\text {TOTAL }} \\
\tilde{F} y_{\text {TOTAL }} \\
\tilde{F} z_{\text {TOTAL }}
\end{array}\right\}=\left\{\begin{array}{l}
\tilde{X} w_{f} \\
\tilde{Y} w_{f} \\
\tilde{Z} w_{f}
\end{array}\right\}+\left\{\begin{array}{l}
\tilde{X}_{A f} \\
\tilde{Y}_{A f} \\
\tilde{Z}_{A f}
\end{array}\right\}+\left\{\begin{array}{c}
\tilde{X}_{M f} \\
\tilde{Y}_{M f} \\
\tilde{Z}_{M f}
\end{array}\right\}
$$

The total moment acting on the projectile in equation (6) comprises the moment due to the standard aerodynamic force $A_{m}$, due to Magnus aerodynamic force $M_{m}$ and the unsteady aerodynamic moment $U A_{m}$ :

$$
\left\{\begin{array}{c}
\tilde{L}_{\text {TOTAL }} \\
\tilde{M}_{\text {TOTAL }} \\
\tilde{N}_{\text {TOTAL }}
\end{array}\right\}=\left\{\begin{array}{c}
\tilde{L}_{A m} \\
\tilde{M}_{A m} \\
\tilde{N}_{A m}
\end{array}\right\}+\left\{\begin{array}{c}
\tilde{L}_{M m} \\
\tilde{M}_{M m} \\
\tilde{N}_{M m}
\end{array}\right\}+\left\{\begin{array}{c}
\tilde{L}_{U A m} \\
\tilde{M}_{U A m} \\
\tilde{N}_{U A m}
\end{array}\right\}
$$

All aerodynamic coefficients are based on Mach number and the aerodynamic angles of attack and sideslip:

$$
\begin{aligned}
& \alpha=\tan ^{-1}\left(\frac{\tilde{w}_{N R F}}{\tilde{u}_{N R F}}\right) \\
& \beta=\tan ^{-1}\left(\frac{\tilde{v}_{N R F}}{\tilde{u}_{N R F}}\right)
\end{aligned}
$$

The total aerodynamic velocity given in equation:

$V_{T}=\sqrt{\tilde{u}_{N R F}^{2}+\tilde{v}_{N R F}^{2}+\tilde{w}_{N R F}^{2}}$

The weight force in no-roll system is:

$$
\left\{\begin{array}{c}
\tilde{X} w_{f} \\
\tilde{Y} w_{f} \\
\tilde{Z} w_{f}
\end{array}\right\}=m g\left\{\begin{array}{c}
-\sin \theta \\
0 \\
\cos \theta
\end{array}\right\}
$$

The aerodynamic force, which acts on the projectile at aerodynamic center of pressure, is: 


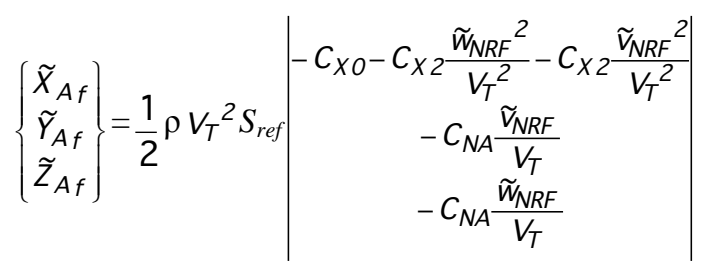

The Magnus, which acts on projectile at the Magnus force center of pressure, is:

$$
\left\{\begin{array}{c}
\widetilde{X}_{M f} \\
\widetilde{Y}_{M f} \\
\widetilde{Z}_{M f}
\end{array}\right\}=\frac{1}{2} \rho V_{T}^{2} S_{r e f}\left|\begin{array}{c}
0 \\
\frac{\widetilde{p}_{N R F} D C_{N P A} \widetilde{w}_{N R F}}{2 V_{T}^{2}} \\
\frac{-\widetilde{p}_{N R F} D C_{N P A} \widetilde{r}_{N R F}}{2 V_{T}^{2}}
\end{array}\right|
$$

The moment due to aerodynamic force is:

$$
\left\{\begin{array}{l}
\tilde{L}_{A m} \\
\widetilde{M}_{A m} \\
\widetilde{N}_{A m}
\end{array}\right\}=\left[\begin{array}{ccc}
0 & 0 & 0 \\
0 & 0 & -R_{\oplus M A C} \\
0 & R_{\oplus M A C} & 0
\end{array}\right]\left[\begin{array}{l}
\tilde{X}_{A f} \\
\tilde{Y}_{A f} \\
\tilde{Z}_{A f}
\end{array}\right]
$$

The moment due to Magnus force is:

$$
\left\{\begin{array}{c}
\tilde{L}_{M m} \\
\widetilde{M}_{M m} \\
\widetilde{N}_{M m}
\end{array}\right\}=\left[\begin{array}{ccc}
0 & 0 & 0 \\
0 & 0 & -R_{\oplus M A X} \\
0 & R_{\oplus M A X} & 0
\end{array}\right]\left[\begin{array}{c}
\tilde{X}_{M f} \\
\tilde{Y}_{M f} \\
\tilde{Z}_{M f}
\end{array}\right]
$$

In addition, for the unsteady moment $U A_{m}$ is:

$$
\left\{\begin{array}{c}
\tilde{L}_{U A m} \\
\tilde{M}_{U A m} \\
\tilde{N}_{U A m}
\end{array}\right\}=\frac{1}{2} \rho V_{T}^{2} D S_{r e f}\left|\begin{array}{c}
\frac{\tilde{p}_{N R F} D C_{L P}}{2 V} \\
\widetilde{q}_{N R F} D C_{M Q} \\
\frac{2}{V}+\frac{C_{M A}}{V} \\
\frac{\tilde{r}_{N R F} D C_{M Q}}{2 V}-\frac{C_{M A}}{V}
\end{array}\right|
$$

The dynamic equations of motion (3-6) are highly nonlinear. Thus, numerical integration is commonly used to obtain solutions to this initial value problem.

\section{MODIFIED PROJECTILE LINEAR THEORY}

To develop the modified projectile linear theory [7], the following sets of simplifications are employed: the axial velocity $\tilde{u}_{N R F}$ can be replaced by the total velocity $V_{T}$ because the side velocities $\tilde{v}_{N R F}$ and $\tilde{w}_{N R F}$ are small.

The aerodynamic angles of attack $\alpha$ and sideslip $\beta$ are small for the main part of the atmospheric trajectory

$$
\alpha \approx \tilde{w}_{N R F} / V_{T}, \beta \approx \tilde{v}_{N R F} / V_{T}
$$

and the projectiles are geometrically symmetrical

$$
\mathrm{I}_{\mathrm{XY}}=\mathrm{I}_{\mathrm{YZ}}=\mathrm{I}_{\mathrm{XZ}}=0, \mathrm{I}_{\mathrm{YY}}=\mathrm{I}_{\mathrm{ZZ}}
$$

Constant aerodynamic coefficients for the most important forces and moments with respect to angle of attack and Mach number are taken into account. Flat-fire and small yaw trajectories are considered so the yaw angle $\psi$ is small:

$$
\sin (\psi) \approx \psi, \cos (\psi) \approx 1
$$

The independent variable is changed from time to dimensionless arclength s, measured in calibers of travel: $s=\frac{1}{d} \int_{0}^{t} V d t$

This technique causes the equations determining the coupled pitching and yawing motion independent of the size of projectile, which turns out to be very convenient in the analysis of free-flight range data.

\section{DIFFERENTIAL EQUATION OF MOTION}

The differential equation governing the angular oscillatory motion for the complete linearized pitching and yawing motion of slightly symmetric projectiles [6] as a function of distance traveled $\mathrm{s}$ is shown below:

$\zeta^{\prime \prime}+(H-i P) \zeta^{\prime}-(M+i P T) \zeta=i P G$

where

$$
\begin{aligned}
& H=\frac{\rho S d}{2 m} C_{L}-\frac{\rho S d}{2 m} C_{D}-\mathrm{K}_{y}^{-2}\left(\frac{\rho S d}{2 m} C_{M Q}\right) \\
& P=\left(\frac{I_{X}}{I_{Y}}\right)\left(\frac{p d}{V}\right) \\
& M=\mathrm{K}_{y}{ }^{-2}\left(\frac{\rho S d}{2 m} C_{M A}\right) \\
& T=\left(\frac{\rho S d}{2 m} C_{L}\right)+\mathrm{K}_{x}{ }^{-2}\left(\frac{\rho S d}{2 m} C_{N P A}\right) \\
& G=\frac{g d \cos \phi}{V^{2}} \\
& \zeta=\alpha+i \beta
\end{aligned}
$$

This differential equation contains all the significant aerodynamic forces and moments that affect the pitching and yawing motion of a spinning or non-spinning symmetric projectile body. The author's definition $\zeta=\alpha+i \beta$ was first chosen by Fowler et al. and was adopted by R. H. Kent. Gunners and engineers usually prefer this definition. The gunner observer looks downrange from a position located just behind the gun. Upward and to the right are always considered as positive directions. It is nature for the gunner to define the $\alpha$ axis as positive upward, the $\beta$ axis as positive to right and the clockwise direction of all rotations as positive for right hand twist rifling.

\section{STATIC AND DYNAMIC STABILITY CRITERIA}

The solution of differential equation (19) tells us that the epicyclic frequencies depend only on the dimensionless roll rate $\mathrm{P}$ and overturning moment $\mathrm{M}$, and are unaffected by any of other aerodynamic forces and moments.

From the definition of an unstable motion, we are led naturally to the concept of static stability:

$\left.\left(P^{2}-4 M\right)\right\rangle 0$

Classical exterior ballistics defines the static stability factor [5] $\mathrm{S}_{\mathrm{g}}$, as:

$$
S_{g}=\frac{I_{X}{ }^{2} p^{2}}{2 \rho I_{Y} S d V^{2} C_{M A}}
$$


Eliminating $\mathrm{P}^{2}$ between equations (26-27), we have:

$4 M\left(S_{g}-1\right)>0$

For statically unstable (spin-stabilized) projectile, $\mathrm{M}>0$ and equation (28) reduces to the classical static stability criterion:

$\left.S_{g}\right\rangle 1$

Equation (26) is a more general result than the (29), because it shows that a statically stable missile $(\mathrm{M}<0)$, is statically stable without spin.

Dynamic stability requires that both damping exponents be negative throughout the projectile's flight. For a nonspinning statically stable missile $(M<0)$ and $P$ is either zero. For finned missiles, the pitch damping moment coefficient is usually negative. The lift and drag force coefficients are both positive, therefore $\mathrm{H}$ is nearly always greater than zero, and the dynamic stability is assured.

The dynamic stability factor $\mathrm{S}_{\mathrm{d}}$ is defined as:

$S_{d}=\frac{2 T}{H}$

and the expressions

$\mathrm{H}>0$

$\frac{1}{S_{g}}\left\langle S_{d}\left(2-S_{d}\right)\right.$

are the generalized dynamic stability criteria for any spinning or non-spinning symmetric projectile flight body.

\section{COMPUTATIONAL SIMULATION}

The constant dynamic flight model [10] uses mean values of the experimental aerodynamic coefficients variations (Table 2).

Table 2. Constant Aerodynamic Parameters for Dynamic Trajectory Flight of the Two Projectile Types

\begin{tabular}{|l|l|}
\hline 105mm Projectile & 120mm Projectile \\
\hline \hline$C_{D}=0.243, C_{L}=1.76$, & $C D=0.14, C L=2.76$, \\
$C_{L P}=-0.0108, C_{M Q}=-9.300$, & $C M Q=-22.300$, \\
$C_{M A}=3.76, C_{Y P A}=0.381$ & $C M A=-15.76$ \\
$C_{N P A}=0.215$ & \\
\hline
\end{tabular}

Initial data for $105 \mathrm{~mm}$ dynamic trajectory model with constant aerodynamic coefficients are:

$\mathrm{x}=0.0 \mathrm{~m}, \mathrm{y}=0.0 \mathrm{~m}, \mathrm{z}=0.0 \mathrm{~m}, \varphi=0.0^{\circ}, \theta=45.0^{\circ}$ and $70.0^{\circ}, \psi=3.0^{\circ}, \tilde{u}=494 \mathrm{~m} / \mathrm{s}, \tilde{v}=0.0 \mathrm{~m} / \mathrm{s}, \tilde{w}=0.0 \mathrm{~m} / \mathrm{s}$, $\tilde{p}=1644 \mathrm{rad} / \mathrm{s}, \tilde{q}=0.0 \mathrm{rad} / \mathrm{s}$ and $\tilde{r}=0.0 \mathrm{rad} / \mathrm{s}$.

The axial spin rate is calculated from

$\tilde{p}=2 \pi V_{T} / \eta D(\mathrm{rad} / \mathrm{s})$

where $V_{T}$ is the total firing velocity $(\mathrm{m} / \mathrm{s}), \eta$ the rifling twist rate at the gun muzzle (calibers per turn), and D the reference diameter of the projectile type $(\mathrm{m})$. are:

In addition, the corresponding initial data for $120 \mathrm{~mm}$ $\mathrm{x}=0.0 \mathrm{~m}, \mathrm{y}=0.0 \mathrm{~m}, \mathrm{z}=0.0 \mathrm{~m}, \varphi=0.0^{\circ}, \theta=45.0^{\circ}$ and $85.0^{\circ}, \psi=8.0^{\circ}, \tilde{u}=318 \mathrm{~m} / \mathrm{s}, \tilde{v}=0.0 \mathrm{~m} / \mathrm{s}, \tilde{w}=0.0 \mathrm{~m} / \mathrm{s}$, $\tilde{p}=0.0 \mathrm{rad} / \mathrm{s}, \tilde{q}=1.795 \mathrm{rad} / \mathrm{s}$ and $\tilde{r}=0.0 \mathrm{rad} / \mathrm{s}$.

The density and pressure are calculated as function of altitude from the simple exponent model atmosphere, and gravity acceleration is taken into account with the constant value $\mathrm{g}=9.80665 \mathrm{~m} / \mathrm{s}^{2}$.

The flight dynamic models of $105 \mathrm{~mm} \mathrm{HE} \mathrm{M1} \mathrm{and} 120$ $\mathrm{mm} \mathrm{HE}$ mortar projectile types involves the solution of the set of the twelve first order ordinary differentials, equations (3)-(6), which are solved simultaneously by resorting to numerical integration using a 4th order Runge-Kutta method, and regard to the 6-D nominal atmospheric projectile flight.

\section{RESULTS AND DISCUSSION}

The flight path trajectories motion with constant aerodynamic coefficients of the $105 \mathrm{~mm}$ projectile with initial firing velocity of $494 \mathrm{~m} / \mathrm{sec}$, initial yaw angle $3 \mathrm{deg}$ and rifling twist rate 1 turn in 18 calibers $(1 / 18)$ at $45^{\circ}$ and $70^{\circ}$ are illustrated in (Fig. 2).

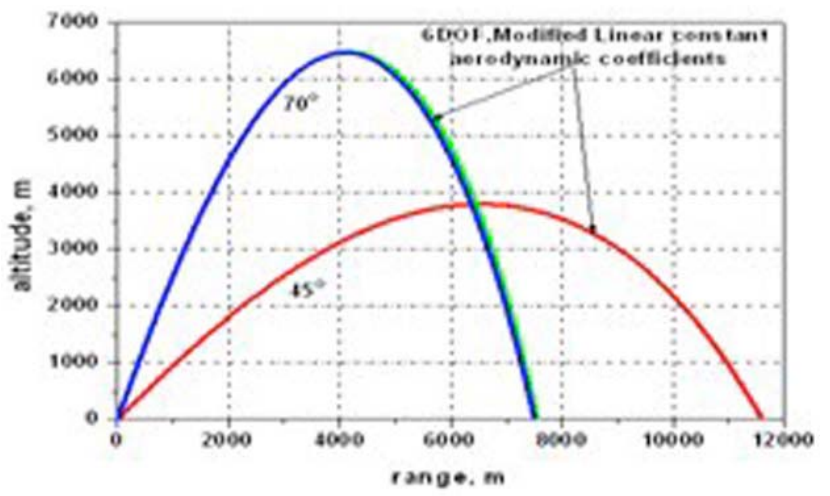

Fig. (2). Impact points and flight path trajectories of 6-DOF and Modified Linear with constant aerodynamic coefficients for 105 $\mathrm{mm}$ projectile.

At $45^{\circ}$ the 6 -DOF model for $105 \mathrm{~mm} \mathrm{M} 1$ projectile, fired at sea-level neglecting wind conditions, gives a predicted range to impact of approximately $11,600 \mathrm{~m}$ and a maximum height at almost $3,600 \mathrm{~m}$. From the results of the modified linear model, the maximum range and the maximum height are almost the same, as shown in (Fig. 2). Also at $70^{\circ}$, the predicted level-ground range of $6-\mathrm{DOF}$ model is $7,500 \mathrm{~m}$ with maximum height at about $6,450 \mathrm{~m}$ and the modified linear trajectory simulation gives the same values.

The mortar projectile of $120 \mathrm{~mm}$ diameter is also examined for its atmospheric constant flight trajectories predictions at pitch angles of $45^{\circ}$ and $85^{\circ}$, with initial firing velocity of $318 \mathrm{~m} / \mathrm{s}$, initial yaw angle $8^{\circ}$ and pitch rate $1.795 \mathrm{rad} / \mathrm{s}$, as shown in (Fig. 3).

At $45^{\circ}$, the $120 \mathrm{~mm}$ mortar projectile, fired at no wind conditions, the 6-DOF trajectory gives a range to impact at $7,000 \mathrm{~m}$ with a maximum height at almost $2050 \mathrm{~m}$. At $85^{\circ}$, the predicted level-ground range is approximately $1,230 \mathrm{~m}$ and the height is $3,950 \mathrm{~m}$. For the same initial pitch angles, the modified linear model's results have satisfactory agreement. (Fig. 3).

In (Fig. 4), static stability factor for the $105 \mathrm{~mm} \mathrm{HE} \mathrm{M1}$ projectile trajectory motion with constant aerodynamic coef- 
ficients is calculated at pitch angles $45^{\circ}$ and $70^{\circ}$, respectively. After the damping of the initial transient motion, at apogee, the stability factor for 45 degrees has increased from 3.1 at muzzle to 23 and then decreased to value of 8 at final impact point. The corresponding flight behavior at $70 \mathrm{de}-$ grees initial pitch angle shows that the transient motion damps out quickly, where the gyroscopic static stability factor has increased from 3.1 to 121 and then decreased to value of almost 6.6 at the impact area.

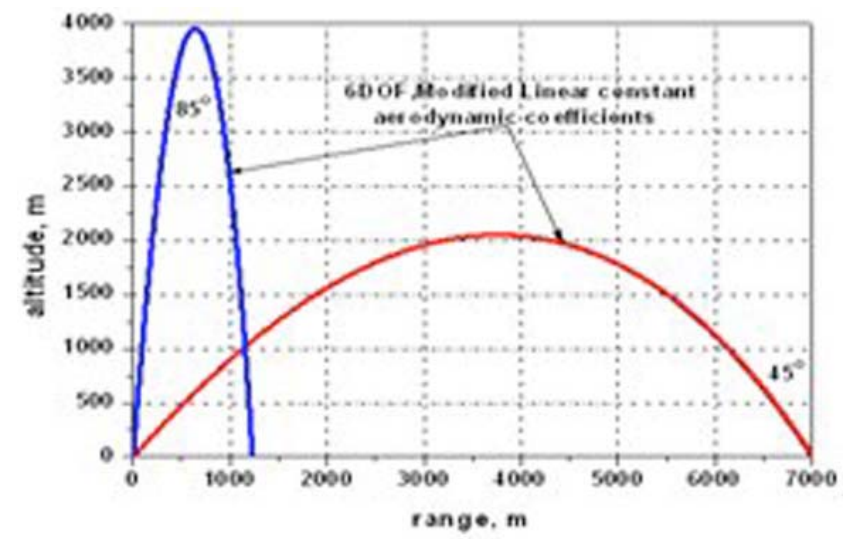

Fig. (3). Flight path trajectories of 6-DOF and Modified Linear with constant aerodynamic coefficients for $120 \mathrm{~mm}$ at quadrant elevation angles of $45^{\circ}$ and $85^{\circ}$.

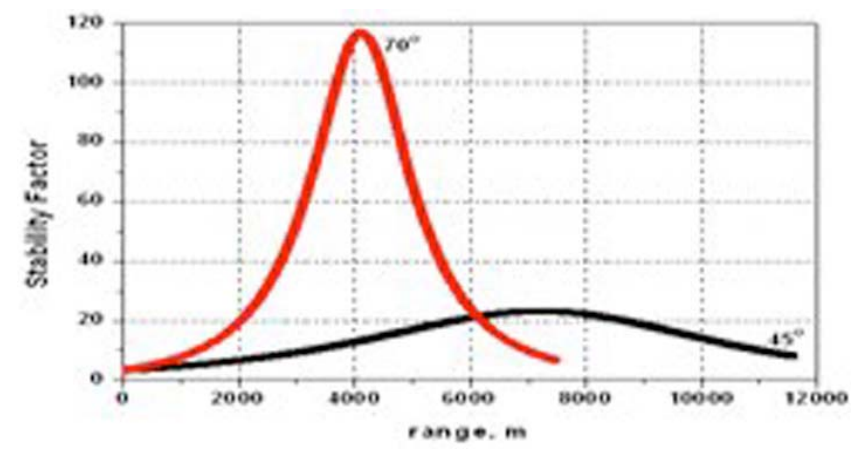

Fig. (4). Comparative static stability variation with constant aerodynamic model at low and high quadrant angles for the $105 \mathrm{~mm}$ projectile.

In (Fig. 5), at 45 and 70 degrees the Mach number was 1.45 at the muzzle, then decrease to 0.6 and 0.3 at the summit of the trajectory and then decrease to values of 0.8 and 0.9 at the impact area, respectively. The constant value of dynamic stability for $105 \mathrm{~mm}$ is 1.095 and lies within the interval $\left(0<S_{d}<2\right)$. So the spin-stabilized $105 \mathrm{~mm}$ projectile is gyroscopically and dynamically stable.

On the other hand the $120 \mathrm{~mm}$ mortar projectile has uncanted fins, and do not roll or spin at any point along the trajectory. Because of that, the static stability is zero, and we examined only the dynamic stability for $120 \mathrm{~mm}$ projectile.

In the modified linear trajectory of the $120 \mathrm{~mm}$ mortar projectile, the constant value of dynamic stability is 0.735 at pitch angles of 45 and 85 degrees. If the dynamic stability factor $S_{d}$, lies within the interval $\left(0<S_{d}<2\right)$, a statically stable projectile is always dynamically stable, regardless of spin. The mortar projectile of $120 \mathrm{~mm}$ belongs in the above interval and $\mathrm{H}>0$, so is dynamically stable.

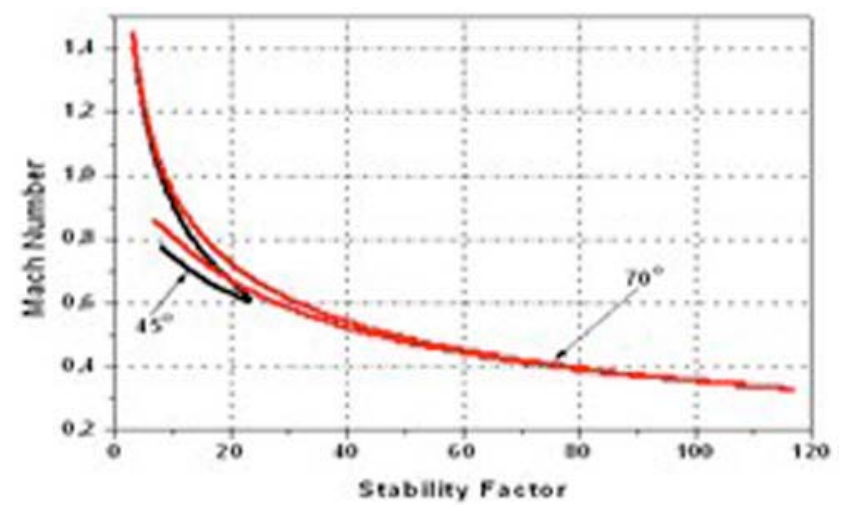

Fig. (5). Comparative stability factor versus Mach number with constant aerodynamic model at low and high quadrant angles for the $105 \mathrm{~mm}$ projectile.

\section{CONCLUSION}

A six degrees of freedom (6-DOF) simulation flight dynamics model is applied for the accurate prediction of short and long-range trajectories for spin and fin-stabilized projectiles. The modified projectile linear theory trajectory reported here should prove useful to estimate flight trajectory's phenomena as static and dynamic stability.

\section{A LIST OF SYMBOLS}

$C_{D 0}$

$C_{D 2}$

$C_{L}$

$C_{L P}$

$C_{M Q}$

$C_{M A}$

$C_{Y P A}$

$C_{N P A}$

$S_{g}$

$S_{d}$

$\bar{x}_{i f}, \bar{y}_{i f}, \bar{z}_{i f}$

$\tilde{u}_{N R F}, \tilde{v}_{N R F}, \tilde{w}_{N R F}$

$\tilde{p}_{N R F}$

$\tilde{q}_{N R F}, \tilde{r}_{N R F}$

$\bar{\theta}, \bar{\psi}$

$\bar{\phi}$

I

$I_{X X}, I_{Y Y}, I_{Z Z}$

$I_{X Y}, I_{Y Z}, I_{X Z}$
= zero-yaw drag aerodynamic coefficient

$=$ yaw drag aerodynamic coefficient

$=$ lift aerodynamic coefficient

$=$ roll damping aerodynamic coefficient

$=$ pitch damping aerodynamic coefficient

$=$ overturning moment coefficient

$=$ Magnus moment coefficient

$=$ Magnus force aerodynamic coefficient

$=$ static stability factor

$=$ dynamic stability factor

$=$ projectile position, $\mathrm{m}$

$=$ projectile velocity components expressed in no-roll-frame, $\mathrm{m} / \mathrm{s}$

$=$ projectile roll rate, $\mathrm{rad} / \mathrm{s}$

$=$ projectiles pitch and yaw rates expressed in no-roll-frame, $\mathrm{rad} / \mathrm{s}$

$=$ projectiles pitch and yaw angles, deg

$=$ projectile roll angle, $\mathrm{deg}$

$=$ projectile inertia matrix

$=$ diagonal components of the inertia matrix

$=$ off-diagonal components of the inertia matrix 


\begin{tabular}{|c|c|}
\hline$V_{T}$ & $=$ total aerodynamic velocity, $\mathrm{m} / \mathrm{s}$ \\
\hline$\rho$ & $=$ atmospheric density, $\mathrm{kg} / \mathrm{m}^{3}$ \\
\hline$D$ & $=$ projectile reference diameter, $\mathrm{m}$ \\
\hline$S_{r e f}$ & $=$ projectile reference area $\left(\pi \mathrm{D}^{2} / 4\right), \mathrm{m}^{2}$ \\
\hline$m$ & $=$ mass of projectile, $\mathrm{kg}$ \\
\hline $\mathrm{t}$ & $=$ time, $\mathrm{s}$ \\
\hline$\alpha, \beta$ & $\begin{aligned}= & \text { aerodynamic angles of attack and } \\
& \text { sideslip, deg }\end{aligned}$ \\
\hline$R_{\oplus M A C}$ & $\begin{aligned}= & \text { distance from the projectile center of } \\
& \text { mass to the center of pressure, } m\end{aligned}$ \\
\hline$R_{\oplus M A X}$ & $\begin{array}{l}=\text { distance from the projectile center of } \\
\text { mass to the Magnus center of pres- } \\
\text { sure, } m\end{array}$ \\
\hline$g$ & $=$ gravity acceleration, $\mathrm{m} / \mathrm{s}^{2}$ \\
\hline
\end{tabular}

\section{REFERENCES}

[1] Fowler R, Gallop E, Lock C, Richmond H. The Aerodynamics of Spinning Shell. London; 1920.

[2] Kent R. An Elementary Treatment of the Motion of a Spinning Projectile About its center of Gravity. USA; 1937.

[3] Nielson K, Synge J. On the Motion of Spinning Shell. USA; 1943.

[4] Kelley J, McShane E. On the Motion of a Projectile with Small or Slowly Changing Yaw. USA; 1944.

[5] Kelley J, McShane E, Reno F. Exterior Ballistics. Denver; 1953.

[6] McCoy R. Modern Exterior Ballistics. Attlen, PA; 1995.

[7] Hainy L, Costello M. Modified Projectile Linear Theory for Rapid Trajectory Prediction. Journal of Guidance, Control, and Dynamics, 2005; Vol.28: No. 5.

[8] Etkin B. Dynamics of Atmospheric Flight, New York; 1972.

[9] Costello M, Anderson D. Effect of Internal Mass Unbalance on the Terminal Accuracy and Stability of a projectile. AIAA Paper; 1996.

[10] Gkritzapis DN, Panagiotopoulos EE, the 2nd International Conference on Experiments / Process / System Modelling / Simulation / Optimization, 2nd IC-EpsMsO. Atmospheric Flight Dynamic Simulation Modelling of Spin-Stabilized Projectiles; July 2007; Athens, Greece; 2007.

(C) Gkritzapis et al.; Licensee Bentham Open.

This is an open access article licensed under the terms of the Creative Commons Attribution Non-Commercial License (http://creativecommons.org/licenses/by-nc/3.0/) which permits unrestricted, non-commercial use, distribution and reproduction in any medium, provided the work is properly cited. 